\title{
Philosophiques
}

\section{Manon Garcia, On ne naît pas soumise, on le devient, Paris, Climats Flammarion, 2018, 272 pages}

\section{Marie-Anne Casselot}

Volume 46, numéro 1, printemps 2019

URI : https://id.erudit.org/iderudit/1062022ar

DOI : https://doi.org/10.7202/1062022ar

Aller au sommaire du numéro

Éditeur(s)

Société de philosophie du Québec

ISSN

0316-2923 (imprimé)

1492-1391 (numérique)

Découvrir la revue

Citer ce compte rendu

Casselot, M.-A. (2019). Compte rendu de [Manon Garcia, On ne naît pas soumise, on le devient, Paris, Climats Flammarion, 2018, 272 pages]. Philosophiques,

46(1), 248-252. https://doi.org/10.7202/1062022ar d'utilisation que vous pouvez consulter en ligne.

https://apropos.erudit.org/fr/usagers/politique-dutilisation/ 
Manon Garcia, On ne naît pas soumise, on le devient, Paris, Climats Flammarion, 2018, 272 pages.

Manon Garcia, dans On ne naît pas soumise, on le devient, attaque de front l'enjeu de la soumission féminine, tabou à la fois philosophique et féministe s'il en est un. D'entrée de jeu, Garcia pose la question provocatrice de la participation des femmes à leur propre soumission: serait-ce une participation active, volontaire, voire voulue? Pour résoudre cette question, Garcia s'attarde, dans cet excellent ouvrage de philosophie féministe, aux effets de la soumission féminine depuis le point de vue des femmes et «dans ce qu'elle a d'aliénant et de séduisant» (p. 24). Ce qui la conduira à une seconde question, non moins provocatrice que la première: serait-ce parce que les femmes tirent du plaisir ou certains avantages à leur soumission qu'elles s'y engagent activement? Quelle situation et quelles motivations sont sous-jacentes à cette participation active?

Selon Garcia, autant les philosophes que les féministes ont fait l'économie d'une étude approfondie de la soumission, ce qui empêche de saisir aujourd'hui toutes les subtilités du phénomène. La philosophie occidentale n'aurait pas étudié les motivations derrière la soumission d'un sujet, que ce soit face à un tyran ou bien à un pouvoir donné. C'est à l'aune du problème de la motivation à la soumission que Garcia réfère rapidement à La Boétie et à Freud comme deux philosophes ayant réellement pensé la soumission, malgré leurs limites théoriques respectives. Le premier a cherché à délimiter les raisons qui poussent un individu à se soumettre à un tyran, tandis que le second suggère une explication psychanalytique du "plaisir pris à sa propre souffrance» dans le masochisme. D'un côté, la soumission est une faute morale tandis que de l'autre, elle est une pathologie. Pour Garcia, les féministes n'ont pas plus résolu «l'énigme de la soumission» (p. I4) que les philosophes, notamment parce qu'elles cherchaient surtout à éviter le piège de l'essentialisme soutenant que la soumission féminine est naturelle à cause de l'infériorité des femmes. Historiquement, la soumission a été "prescrite comme un comportement normal, moral et naturel de la femme» (p. I5); dans ce contexte, les femmes étaient des victimes passives de la domination masculine.

Garcia propose pour sa part qu'étudier la soumission, d'un point de vue féministe, ne doit pas présupposer un déterminisme du destin des femmes - elles ne sont "pas des victimes, coupables, passives ou perverses » (p. I9). Réfléchir à la soumission féminine implique de "décrire une expérience vécue sans pour autant considérer cette expérience comme absolue, naturelle et nécessaire pour être une femme»(p. 2I). La perspective de Garcia considère plutôt que "la soumission c'est l'action ou la situation de celui ou celle qui se soumet»(p. 3I). Lorsqu'elle configure sa définition d'usage de la soumission, Garcia affirme qu'il y a eu décision consciente, dans la soumission, "de ne pas agir contre le pouvoir qui s'exerce sur soi » 
(p. 3I). La philosophe française distingue deux volontés possibles à l'œuvre dans la soumission: une volonté positive d'être soumis ou bien une volonté passive s'assimilant à de la résignation et à l'absence de résistance. Pour son projet, Garcia soutient donc qu' "étudier la soumission des femmes consiste à étudier l'action ou la situation des femmes lorsqu'elles sont partie prenante d'un rapport de domination auquel elles ne résistent pas» (p. 34).

Outre la réarticulation de la soumission en termes de participation active plutôt qu'en termes d'absence de résistance, l'intérêt principal du livre réside dans l'interprétation et la vulgarisation de la pensée de Simone de Beauvoir pour comprendre la soumission comme une prescription sociale inéluctable pour les femmes. Faisant fi d'une supposée «essence féminine soumise » Garcia explique que la soumission est le comportement prescrit aux femmes: si elles se soumettent effectivement, elles en tirent plusieurs bénéfices (tels que le statut socio-économique, la désirabilité, la valeur morale, etc.).

Afin de contextualiser Beauvoir, Garcia procède à une synthèse des influences de Martin Heidegger et de Jean-Paul Sartre pour expliquer les racines existentialistes du concept de situation. Il s'agit effectivement d'une bonne introduction à la pensée existentialiste, mais la démonstration ralentit à certains égards l'argument principal de Garcia. Cela dit, le concept de situation est central au projet du Deuxième sexe de Beauvoir, et Garcia ne peut en faire l'économie. La situation recouvre le contexte social, économique et politique dans lequel un individu existe, dont il peut partiellement se déprendre à travers ses projets et ses actions. Ainsi, appliqué aux femmes, «le concept de situation permet à la fois de décrire la réalité de l'infériorité des femmes par rapport aux hommes et de l'historiciser, c'est-à-dire de montrer qu'il n'y a rien de naturel et donc de fixé dans cette infériorité » (p. 7I). Garcia peut alors avancer que, dans la société patriarcale, la norme sociale prescrite de la féminité est la soumission (p. I25).

Pour Garcia, la perspective beauvoirienne empêche de "penser l'individu en dehors de sa situation sociale» (p. 85), ce qui revient à dire que la soumission ne peut se comprendre comme une caractéristique individuelle ou fixe. La méthodologie originale de Beauvoir s'enracine dans la multiplication des expériences vécues singulières des femmes, à travers le grand nombre de témoignages, de récits littéraires, de journaux intimes présentés dans Le deuxième sexe. Garcia qualifie la méthodologie beauvoirienne comme une démarche inductive à la fois ascendante et descendante. C'est une méthodologie ascendante parce que Beauvoir part d'expériences singulières multiples, en fait ressortir les similarités pour enfin attester d'une régularité dans l'expérience de la soumission féminine. Ensuite, c'est une méthode descendante parce qu'elle permet de «comprendre la façon dont la situation générale de la femme influe sur les expériences vécues des femmes particulières" (p. I32).

Avec Beauvoir, Garcia retrace la structure de la soumission féminine à même la catégorie de l'Autre et le processus d'objectification à l'œuvre dans 
la dialectique hégélienne du maître et de l'esclave. Les femmes sont «bloquées dans une altérité absolue parce qu'elles [sont] objectifiée[s] par les hommes » (p. I 58 ). Garcia excelle dans son explication de l'objectification comme élément constitutif de la soumission féminine. Dans leur réduction à un statut d'objet désirable pour le regard masculin, les femmes intériorisent que la féminité est synonyme de soumission:

La situation des femmes est indissociable de l'objectification que le regard masculin leur fait subir, et la domination masculine permet à cette objectification de passer inaperçue et de faire apparaître la soumission des femmes non pas comme le résultat de la domination des hommes mais comme leur condition objective (p. I 59).

Toutefois, c'est dans le chapitre "Le corps-objet de la femme soumise » que la maîtrise de Garcia se dévoile entièrement. L'attrait résolument phénoménologique de son livre s'y fait ressentir, car elle illustre la spécificité de l'expérience corporelle telle qu'elle est vécue par les femmes. Pour Garcia, le corps féminin est un corps social avant d'être un corps vécu. C'est-à-dire qu'une femme expérimente son corps comme vu, perçu et désiré par autrui avant d'en vivre individuellement toutes les possibilités. À la puberté, la jeune fille se découvre vue et désirée par autrui avant de connaître elle-même son corps. Ce sont "des corps-pour-autrui avant même d'être des corpspour-soi »(p. I 87). L'identité des femmes serait ainsi étroitement reliée à leur apparence physique, à leur corporalité, et c'est pourquoi Garcia insiste sur la dimension sociale du corps qui structure la situation des femmes en ce qu'elles sont destinées à se soumettre (p. I66). Ici, l'analyse de Garcia rejoint celle d'Iris Marion Young dans Throwing Like a Girl (I980) ou encore la lecture de Gail Weiss sur l'injonction oppressive de l'image corporelle pour les femmes dans Body Images: Embodiment as Intercorporeality (I999). L'expérience corporelle des femmes dénote l'ambiguité fondamentale de leur expérience vécue entre liberté et aliénation; elles sont toujours sous la menace permanente de l'objectification, ce qui entraîne en retour la soumission pour tenter d'y échapper (p. I75). Dans ce processus d'objectification, la femme perd son corps comme étant sa propre prise sur le monde au profit d'un corps comme objet désirable pour les hommes. Processus qui est par ailleurs corollaire de celui par lequel le corps biologique devient le support des significations et des valeurs sociales; dans un contexte patriarcal, le corps des femmes «n'est que le support d'un destin qui est constitué socialement et qui est ensuite naturalisé dans le corps» (p. I7I).

C'est à ce point que l'œuvre de Garcia se complexifie; dans son explication du plaisir pris à la soumission, la philosophe française note qu'il y a un pouvoir dans la passivité de la soumission féminine. Se soumettre au regard masculin, accepter son statut d'objet de désir, c'est un moyen de se réapproprier son corps pour soi. En se faisant consciemment objet de désir, la femme s'active dans la passivité même de la soumission. Malgré l'inéluctabilité de la domination patriarcale, les femmes retrouvent un semblant de 
pouvoir sur leur corps, elles reprennent le contrôle de leur prise sur le monde dans leur désirabilité même. Enfin, Garcia insiste sur le fait que "[l]e plaisir pris dans la soumission naît de la situation spécifique de la femme» (p. I9I), renvoyant ainsi à une soumission qui n'est jamais prédéterminée ni immuable.

Toutefois, le pouvoir érotique qu'elle retire de se soumettre est toujours voué à l'échec car il est la manifestation d'une dépendance totale au regard et au désir masculin (p. I93). La femme soumise se soumet totalement, elle met en jeu son identité et sa valeur dans un besoin de validation par les hommes. Il y a bel et bien des "délices dans la soumission ", tels qu' " esquiver le coût de la liberté", une "démission de soi» dans l'autre, un "plaisir individuel à choisir l'altérité » (p. 210). Garcia, suivant Beauvoir à la trace, souligne aussi que la soumission se manifeste dans les phénomènes du dévouement et de l'amour-abdication. Dans ces phénomènes, il y a démission de soi pour soutenir l'autre, «une forme de pouvoir dans l'anéantissement de soi» (p. 204). Même si, au cœur de cette démission de soi se trouve une exigence radicale envers l'autre: la personne soumise revendique un pouvoir sur l'autre, "une exigence ancrée dans le sacrifice de soi » (p. 20I).

Enfin, Garcia conclut son ouvrage en affirmant que les femmes font, depuis leur situation, un "calcul coûts-bénéfices " entre la conformité aux normes de la féminité soumise et les «risques de la liberté ». Il est donc bien question pour les femmes d'un choix de la soumission, et non pas d'une supposée essence naturelle ou innée. Si la soumission féminine semble malgré tout être un destin, c'est parce qu'elle apparaît comme «la seule stratégie apparemment disponible à la femme pour devenir souveraine et pour acquérir une forme de maîtrise de soi et du monde qui a à voir avec l'autonomie» (p. 206). Fait remarquable, Garcia n'adopte jamais un ton moralisateur envers les femmes qui se soumettent et qui en tirent du plaisir. Toujours, elle entrelace son argumentaire avec des extraits tirés du Deuxième sexe, tout en évitant les écueils dogmatiques dans lesquels Beauvoir elle-même est tombée.

On ne naît pas soumise, on le devient est un ouvrage qui fera date en phénoménologie féministe, pour son propos original sur la soumission autant que pour sa synthèse de la pensée de Simone de Beauvoir. On retrouve des lignes excellentes, voire rafraîchissantes, sur la soumission féminine venant ancrer le projet dans la visée transformatrice de la philosophie féministe en général. Malgré tout, on ressent une certaine précaution prise par l'auteure dans ses nombreux détours théoriques avant d'entrer dans le vif du sujet de la soumission féminine dans les premiers chapitres. De façon évidente, Garcia maîtrise incontestablement son sujet: les quelques plaidoyers pour l'utilité d'une réflexion sur la soumission féminine semblent alors superflus. Cette perception provient sûrement de ce qui semble être l'objectif double de l'ouvrage - à la fois vulgarisation d'un enjeu philosophique et contribution originale contemporaine. 
Bien que Garcia se penche sur l'apport de Catherine MacKinnon à propos du lien structurel entre hétérosexualité et domination, et Gayatri Spivak sur la difficulté des subalternes à parler de leur expérience vécue, l'absence d'autres penseures féministes contemporaines est criante, notamment en ce qui concerne l'enjeu du consentement. Le choix de rester très près de Beauvoir est certes louable, mais plusieurs théoriciennes féministes contemporaines ont soulevé des enjeux limitrophes à la soumission. On pense notamment à Colette Guillaumin qui a réfléchi avec brio aux effets matériels et psychologiques de l'appropriation masculine sur les femmes. Toutefois, l'insistance sur Beauvoir se fait ressentir notamment dans l'absence d'étude approfondie de la notion de consentement. L'originalité d'On ne naît pas soumise, on le devient réside dans ce qu'il nous fait comprendre qu'il y a une part de volonté active et de plaisir dans la soumission féminine, et que cet état de fait provient de la situation ambiguë des femmes dans la société patriarcale. C'est une relation de non-résistance, de résignation, de reprise de pouvoir illusoire dont les femmes tirent des bénéfices matériels et moraux. Or le contexte actuel dans lequel s'inscrit cette œuvre aurait demandé une plus grande attention à l'enjeu du consentement tel qu'il est présentement vécu par les femmes au $\mathrm{XxI}^{\mathrm{e}}$ siècle. Si Garcia mentionne du bout du clavier le moment Moi Aussi, elle n'approfondit pas les ramifications complexes entre le consentement, la violence subie et les tentatives de résistance des femmes. La situation actuelle des femmes est toujours ambiguë et elle a évolué depuis Le deuxième sexe. "Le consentement des femmes à leur propre soumission» (p. 242) prend aujourd'hui un tout autre visage, subtil et complexe, dont on aurait attendu une description contemporaine en conjonction avec l'analyse beauvoirienne qu'exécute Garcia.

Cela étant, On ne naît pas soumise, on le devient s'avère une remarquable contribution sur la complexité de la soumission féminine autant qu'une excellente synthèse de la pensée de Simone de Beauvoir. Écrit dans un style limpide et accessible, c'est un ouvrage de philosophie féministe à mettre entre plusieurs mains.

MARIE-ANNE CASSELOT

Université Laval

\section{Markus Gabriel, Le néo-existentialisme: penser l'esprit humain après l'échec du naturalisme, Québec, Presses de l'Université Laval, 2019, 179 pages.}

Le présent ouvrage est la reprise augmentée et accompagnée de trois commentaires inédits d'une conférence prononcée notamment à l'Université Laval à l'invitation de la Chaire de philosophie dans le monde actuel. Gabriel y développe une objection et une solution de rechange au naturalisme réductionniste. 